\title{
A!
}

This is an electronic reprint of the original article.

This reprint may differ from the original in pagination and typographic detail.

Havu, Paula; Torsti, T.; Puska, M.J.; Nieminen, R.M.

\section{Conductance oscillations in metallic nanocontacts}

Published in:

Physical Review B

DOI:

10.1103/PhysRevB.66.075401

Published: 01/08/2002

Document Version

Publisher's PDF, also known as Version of record

Please cite the original version:

Havu, P., Torsti, T., Puska, M. J., \& Nieminen, R. M. (2002). Conductance oscillations in metallic nanocontacts.

Physical Review B, 66(7), 1-5. [075401]. https://doi.org/10.1103/PhysRevB.66.075401

This material is protected by copyright and other intellectual property rights, and duplication or sale of all or part of any of the repository collections is not permitted, except that material may be duplicated by you for your research use or educational purposes in electronic or print form. You must obtain permission for any other use. Electronic or print copies may not be offered, whether for sale or otherwise to anyone who is not an authorised user. 


\title{
Conductance oscillations in metallic nanocontacts
}

\author{
P. Havu, T. Torsti, M. J. Puska, and R. M. Nieminen \\ Laboratory of Physics, Helsinki University of Technology, P.O. Box 1100, FIN-02015 HUT, Finland
}

(Received 12 April 2002; published 1 August 2002)

\begin{abstract}
We examine the conductance properties of a chain of $\mathrm{Na}$ atoms between two metallic leads in the limit of low bias. Resonant states corresponding to the conductance channel and the local charge neutrality condition cause conductance oscillations as a function of the number of atoms in the chain. Moreover, the geometrical shape of the contact leads influences the conductivity by giving rise to additional oscillations as a function of the lead opening angle.
\end{abstract}

DOI: 10.1103/PhysRevB.66.075401 PACS number(s): 73.21.Hb, 73.40.Cg, 73.63.Nm, 73.63.Rt

\section{INTRODUCTION}

The prospects to fabricate molecular devices, extending from a few atoms to $10-\mu \mathrm{m}$-long carbon nanotubes, have inspired an active research field. ${ }^{1}$ The most modern experimental, theoretical, and computational methods are used to investigate and model ultimate-size transistors, switches, gates, and memory devices as well as their coupling with the rest of the electronic circuit. In this work we concentrate to chains of $\mathrm{Na}$ atoms as ultimate conductors. Similar atomicscale metallic nanowires have been produced from $\mathrm{Au}$ by a scanning tunneling microscope $\mathrm{e}^{2,3}$ and by the mechanically controllable break junction technique. ${ }^{4}$ The formation of $\mathrm{Na}$ nanowires has been investigated by first-principles calculations $^{5-7}$ and the ensuing conductances have been compared with experiments. ${ }^{7}$

The conductance of a nanowire shows nonmonotonic behavior as a function of its length. However, the role of the contact leads cannot be ignored, as shown by Sim et al. ${ }^{8}$ and Yeyati et al. ${ }^{9}$ The purpose of this paper is to study the dependence of the conductance not only on the length but also on the shape of the contacts. We show that the shape of the contact, i.e., the bluntness of the STM tip, influences the conductance in an oscillatory way.

Yeyati et al. ${ }^{9}$ recognized the importance of resonance states to the conductance of sharp leads. These resonances exist only at sharp tips, not at blunt ones. In the case of monovalent atoms, such as $\mathrm{Na}$ or $\mathrm{Au}$, the appearance of a resonance at the Fermi level can explain the first quantized conductance step as a multiple of $2 e^{2} / h$. Sim et al. ${ }^{8}$ showed that the charge neutrality required for the $\mathrm{Na}$ chain and the tips of the contacts leads to an even-odd behavior in the conductance as a function of the number of atoms in the chain. This is because for an odd number of atoms the resonance is half-occupied whereas for an even number of atoms the resonance is fully occupied, leading to a low density of states at the Fermi level.

As Sim et $a l .^{8}$ we see the even-odd behavior for lead tips. In addition, the conductance oscillates as a function of the lead opening angle. This results from the change in the angular character of electrons screening the tip regions of the leads.

\section{MODEL}

As in Ref. 8, we model the nanocontact by a chain of $N$ Na pseudoatoms. ${ }^{10}$ To enable a systematic study of the shape effects, we model the leads using the "stabilized jellium" model, ${ }^{11}$ where leads of discrete ions have been smeared out to continuous positive charge distributions and a constant potential-energy term has been added to stabilize the Na bulk electron density. The computational geometry is depicted in Fig. 1. The positive background charge of each cone corresponds to $M$ electrons. The constant density $n_{+}$of the positive background is determined from the $\mathrm{Na}$ valence electrondensity parameter $r_{s}=3.93$ a.u. $\left[n_{+}=3 /\left(4 \pi r_{s}^{3}\right)\right]$. Near the atom chain, the radius of the cones is $r_{s}$ and the larger radii are determined by the number of electrons in the cones, which can be varied at will. We solve the electronic states of the system using the density-functional theory within the local-density approximation ${ }^{12}$ assuming the limit of zero external bias.

The system has cylindrical symmetry, so that the problem is computationally two dimensional. In the middle of the atom chain there is a mirror plane perpendicular to the symmetry axis. The electronic wave functions are required to be even or odd with respect to the mirror plane. Naturally, the Poisson equation involved in the self-consistent loop is solved by requiring mirror symmetry for the Coulomb potential. On the other boundaries, i.e., on a plane parallel to the mirror plane but far outside the jellium cone and on a cylinder surface far from the axis, the wave functions are required to vanish. The numerical solutions of the density-functional equations are obtained by a real-space multigrid scheme. ${ }^{13}$

In the case of a chain of $\mathrm{Na}$ atoms there is only one conducting eigenchannel contributing to the electron transport at the Fermi level. Then the mirror symmetry and the

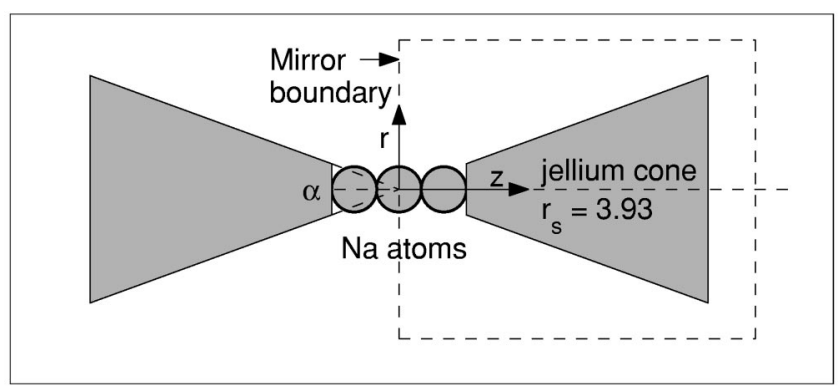

FIG. 1. Geometry of the present model. Na pseudoatoms are located between two jellium cones. The cone angle $\alpha$ can be varied continuously. The dotted line gives the boundaries of the calculation cell. For the details, see also the text. 
Friedel sum rule give the conductance $G$ in the zero-bias limit as ${ }^{14}$

$$
G=\frac{2 e^{2}}{h} \sin ^{2}\left[\frac{\pi}{2}\left(N_{e}-N_{o}\right)\right],
$$

where $N_{e}$ and $N_{o}$ are the numbers of electrons in the evenand odd-parity states, respectively. In an infinite system, $N_{e}$ and $N_{o}$ are calculated by integrating the density of states up to the Fermi level. The states are assumed to have the spin degeneracy and to be occupied accordingly. Then the difference of $N_{e}$ and $N_{o}$ arises only from the electrons of the conducting eigenchannel, because only their wave functions may have a finite value at the mirror plane. In our model the conducting eigenchannel corresponds to the quantum number $m=0$ of the angular momentum around the symmetry axis.

The states with a finite value at the mirror plane $(m=0)$ span an energy region starting somewhat below the Fermi level. They are strongly localized at the chain atoms and the lead tips. These resonance states, as occupied up to the Fermi level, have to participate in neutralizing the positive charge of the atom chain and the tips of the leads. According to the calculations by Sim et al. ${ }^{8}$ for $\mathrm{Na}$ atom chains between two $\mathrm{Na}$ (111) tips, the neutrality requirement leads for an odd number $N$ of $\mathrm{Na}$ atoms to a half-filled resonance in the conduction eigenchannel. This means that the Fermi level is at the resonance state, which is filled in a spin-compensated manner totally by one electron. As a result, the difference $N_{e}-N_{o}$ is odd and the conductance is close to $2 e^{2} / h$. For an even $N$ the Fermi level is off the resonance and the conductance will be less than unity. Below we will show that also electron states that do not correspond to the conducting eigenchannel $(m>0)$ participate in the charge neutralization in the tips, modifying the conductance behavior.

In order to study the convergence of the results as a function of the system size we have performed calculations with $M=200$ and 300 electrons in each jellium cone. The finite size results in a discrete eigenvalue spectrum that is smoothed using the Fermi-Dirac broadening with a finite temperature when occupying the electron states. The broadening should be larger than the single electron level spacing but smaller than the distance between the resonances. The level spacing decreases as $\sim M^{-2 / 3}$ so that we can decrease the temperature with increasing $M$ and obtain sharper resonance structures. For 200 electrons we have chosen the temperature of $600 \mathrm{~K}$, which was used also by Sim et al. ${ }^{8}$ for cones of 60 and 95 electrons. In the case of 300 electrons in a cone we choose the temperature of $400 \mathrm{~K}$.

\section{RESULTS}

Our main result is the finding that the conductance of a chain of atoms depends on the cone angle of the leads. In order to understand the reasons we first discuss the changes in the electronic structure in terms of such integrated quantities as the electron density and the local density of states (LDOS). Thereafter the conductance, which reflects changes in the electronic structure on a microscopic level, is given.
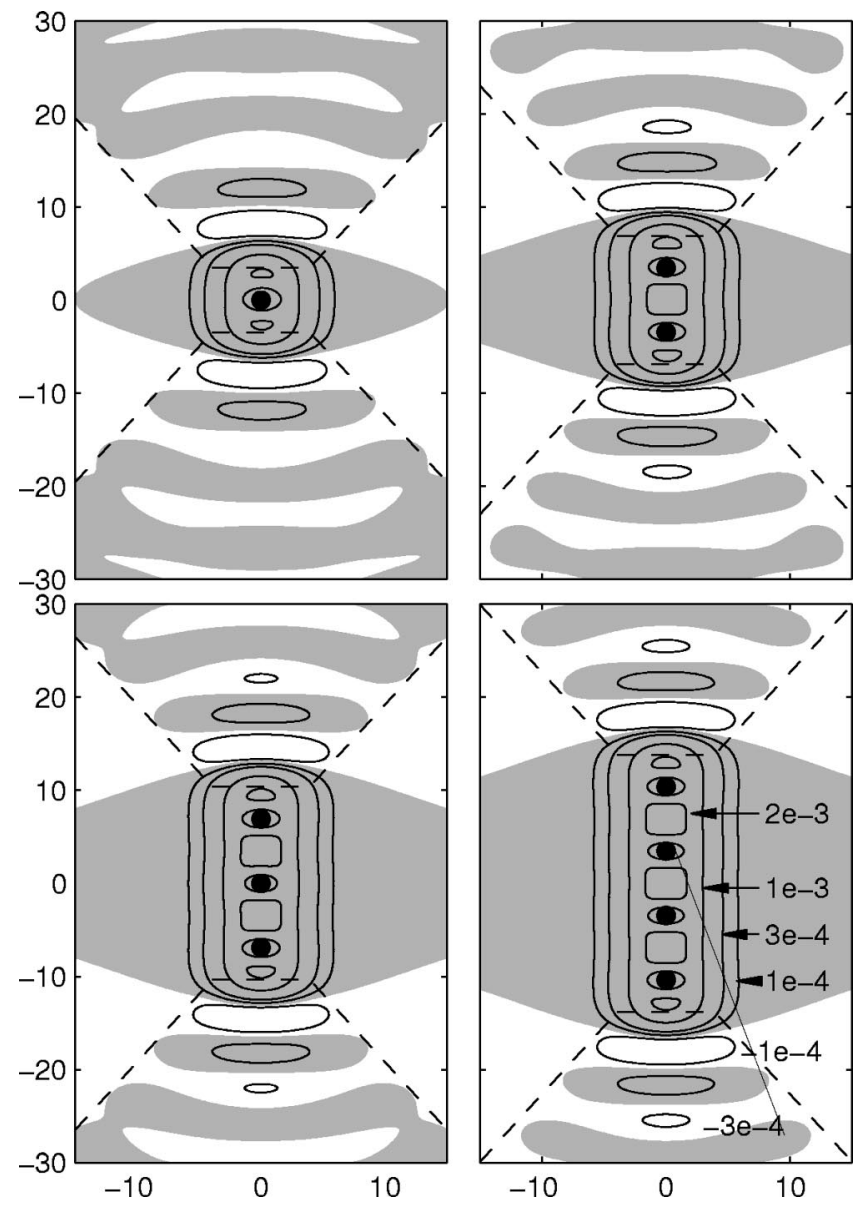

FIG. 2. Electron densities induced by chains of one, two, three, and four pseudoatoms between two jellium leads. The cone angle is $69^{\circ}$ and each of the cones contains 300 electrons. The density values of the contours are given in atomic units $\left(a_{o}^{-3}\right)$. Areas of positive induced density are shown as gray and the ionic positions as black circles.

Figure 2 shows the electron density induced by chains of one to four $\mathrm{Na}$ atoms between two jellium leads containing 300 electrons each. The cone angle is $69^{\circ}$. The induced density is calculated as the difference between the whole system and the corresponding system consisting of the leads only. In accordance with the calculations ${ }^{8}$ having 95 pseudoatoms as leads, the main part of the induced density is localized at the atom chain and the density decays rapidly in the cones. This indicates convergence as a function of the system size. For an even $N$ the electron density is slightly more localized than in the case of an odd $N$ because for an even $N$ strong resonance states are below the Fermi level and are fully occupied. The density of states (DOS) of the system with two $\mathrm{Na}$ atoms is shown in Fig. 3. It is obtained from the discrete energy-level spectrum given at the bottom of the figure by substituting the lines by Lorentzians, the widths of which correspond to the temperature used in the self-consistent electronic structure calculations. We see that around the Fermi level the single electron level spacing is small in comparison with distances between the strong DOS peaks. The latter are related to the $m=0$ resonance structure (the $m$ $=0$ eigenenergies are given in the figure as long vertical 


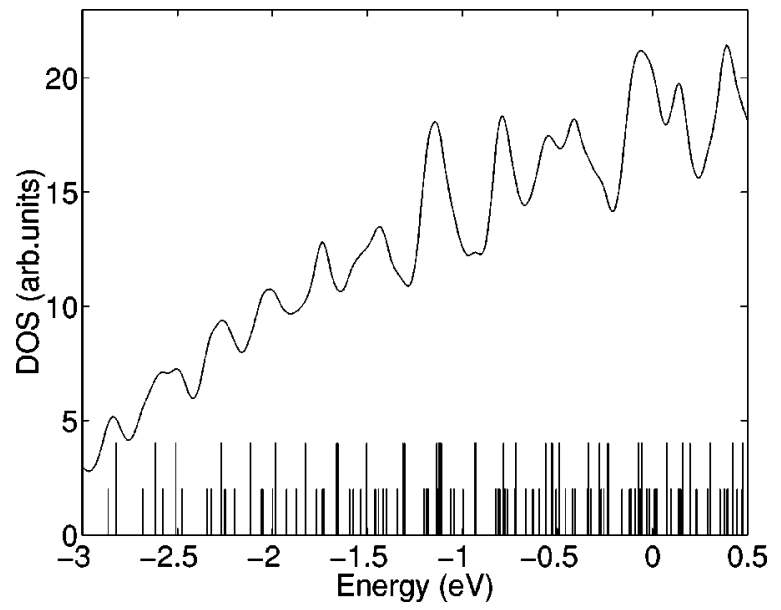

FIG. 3. Chain of two Na atoms between two jellium leads with the cone angles of $69^{\circ}$. The density of states (DOS) is given with respect to the Fermi level (energy zero). The single electron eigenenergies are marked with bars at the bottom of the figure. The $m=0$ states are denoted by longer bars than the $m>0$ states.

lines). It can be seen that the region of peaks with a larger amplitude reaches about $1 \mathrm{eV}$ below the Fermi level.

The character of the electron density near the tips of the leads changes as the cone angle increases. This is demonstrated in Fig. 4 that shows the total electron density and its $m=0$ state component for three different cone angles. The densities are integrated over a plane perpendicular to the cylinder axis and are given as a function of the position $z$ along the axis. Naturally, the total electron density increases close to the tip as the cone angle increases. But it is important to note that the $m>0$ electron states contribute most of the

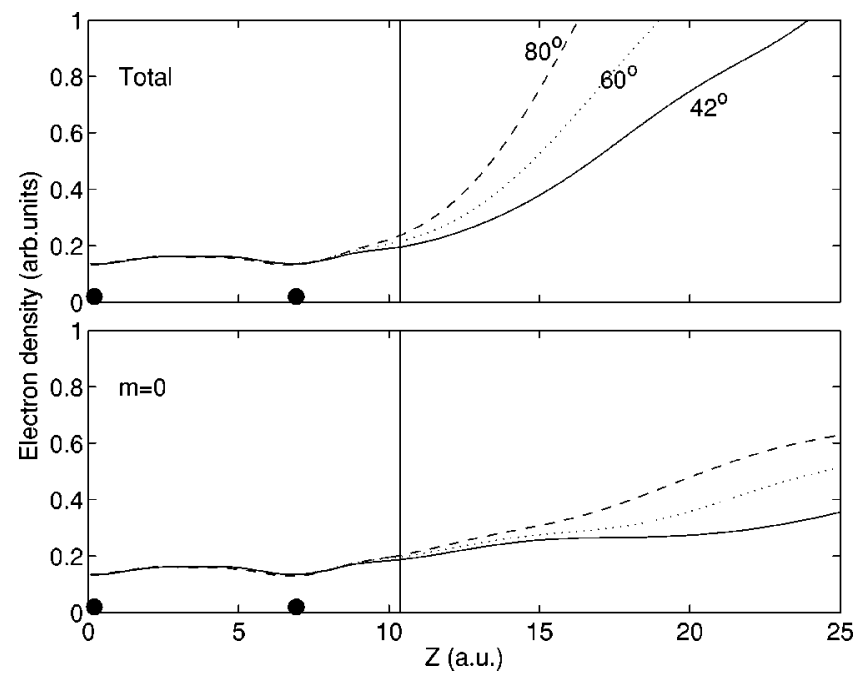

FIG. 4. Chain of two Na atoms between two jellium leads. The electron density integrated over the plane perpendicular to the cylinder axis as a function of the position $z$ along the axis is given. The upper panel shows the total density whereas the lower panel gives the contribution due to the $m=0$ states, only. Densities for the three cone angles of $42^{\circ}, 60^{\circ}$, and $80^{\circ}$ are given by solid, dotted, and dashed lines, respectively. The vertical line marks the jellium edge and the black circles denote the $\mathrm{Na}$ atom positions.
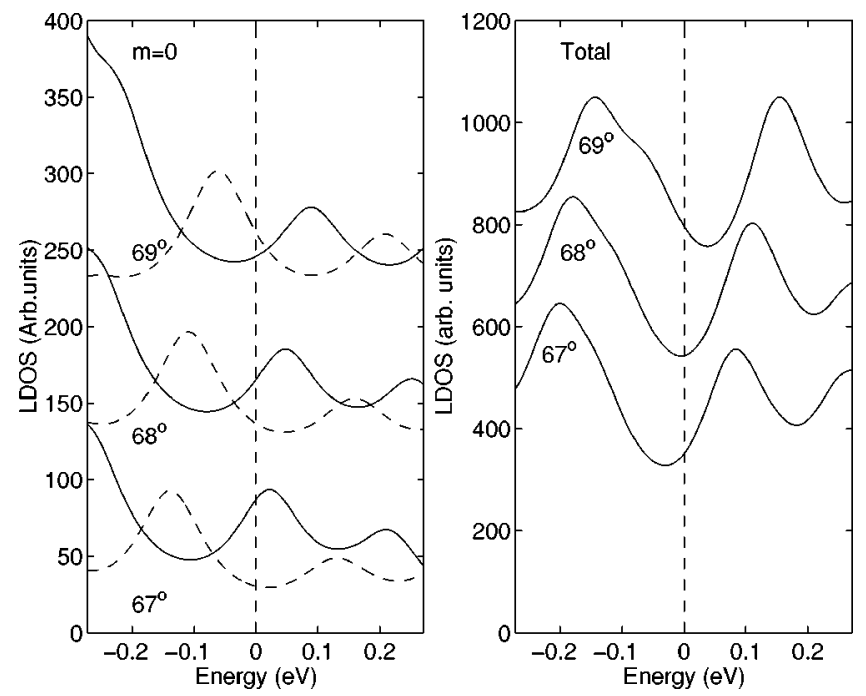

FIG. 5. Chain of two Na atoms between two jellium leads. The local density of states (LDOS) near the Fermi level (energy zero) is given for different cone angles. The left panel shows the the even (solid line) and odd (dashed line) $m=0$ LDOS's calculated for the atom chain between the jellium edges. The right panel shows the total LDOS's calculated for the atom chain and the tips of the leads to the depth of $r_{s}$ from the jellium edge. The LDOS's corresponding to the two uppermost angles are shifted in steps of 100 and 200 units in the left and right panels, respectively.

density increase. As a result, the $m=0$ resonance states feel an increased Coulomb repulsion and are pushed upwards in energy. For the same reason, also other states with low $m$ $(>0)$ values rise in energy, but to a lesser extent.

The shift of the $m=0$ states to higher energies can be seen in the LDOS at the $\mathrm{Na}$ atom chain. We calculate the LDOS similarly to the DOS discussed above, but now the area of a Lorenzian peak is the density of the state in question integrated over a volume limited by two planes perpendicular to the symmetry axis. In the left panel of Fig. 5 we show the LDOS of the even and odd $m=0$ states for the cone angles of $67^{\circ}, 68^{\circ}$, and $69^{\circ}$. The integration is limited to the atom chain, i.e., between the planes at the central jellium edges. The LDOS of the chain has a structure similar to the result of the atomistic lead calculations. ${ }^{8}$ When we open the cone angle, the $m=0$ states are shifted, as discussed above, to higher energies. The conductance of the chain is close to $2 e^{2} / h$ when the Fermi level is located on top of a peak of the $m=0$ LDOS. The left panel of Fig. 5 indicates that the conductance varies rapidly as a function of the cone angle. The right panel of Fig. 5 gives the total LDOS calculated by including the tips of the cones into the integration; the limiting planes are at the depth of $r_{s}$ from the central jellium edges. We see that in this volume, which is important for the charge neutrality of the atom chain, the $m=0$ features of the left panel are hardly visible. The change of this total LDOS is also less dramatic than that of the $m=0$ LDOS at the atom chain.

On the basis of Figs. 4 and 5 we can conclude that the $m>0$ states have an important contribution to the local charge neutrality and influence the conductance of the chain. This is seen in Fig. 6 that gives the conductances of the 


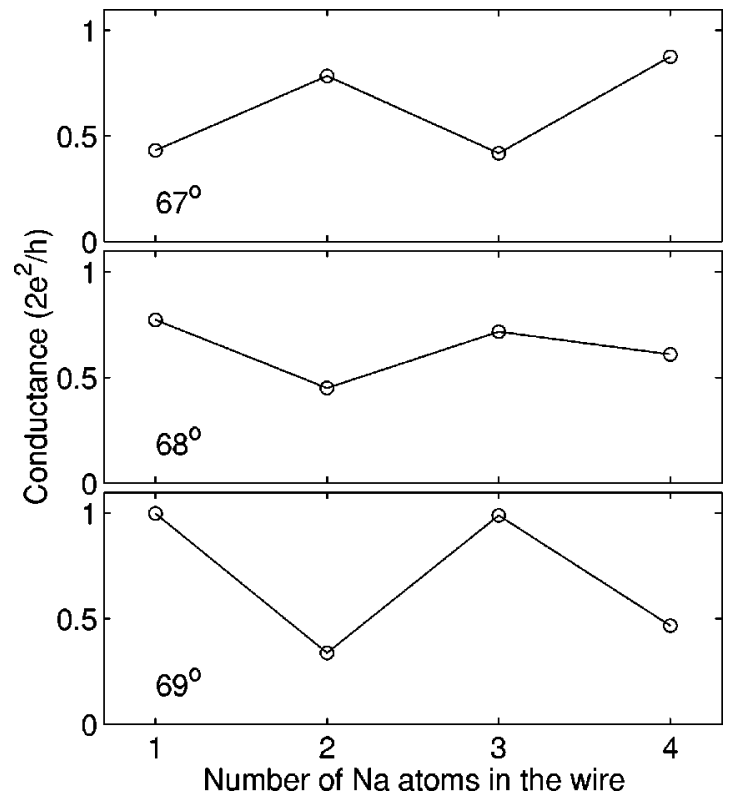

FIG. 6. Conductances of the chains of one, two, three, and four $\mathrm{Na}$ atoms between two jellium leads. The conductances are shown for three different jellium cone angles. The number of electrons in each cone is 300 .

chains of one to four $\mathrm{Na}$ atoms corresponding to these three cone angles. The even-odd oscillation as a function of the number of chain atoms is clear as well as its gradual phase change.

Finally, Fig. 7 shows the conductance for the $N=3$ chain as a function of the cone angle. Results for system sizes corresponding to 200 and 300 electrons in each jellium cone are given. It can be seen that for both system sizes the conductance is a regularly oscillating function of the cone angle. The temperature is closely connected to the amplitude of the oscillations so that a lower temperature gives stronger oscillations. If we choose a high enough temperature, the oscillation as function of angle disappears but so does the oscillation as function of $N$. The number of oscillations seems to increase as the system size increases. This is because the resonance structure becomes more resolved including higher

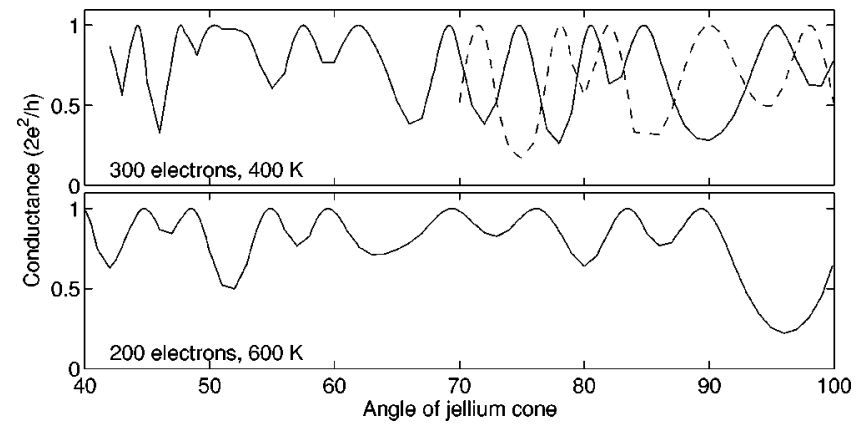

FIG. 7. Chain of three Na atoms between two jellium leads. The conductance is given as function of cone angle for systems of 200 and 300 electrons in one lead. The temperature used in occupying the one-electron states is $600 \mathrm{~K}$ for the smaller system and $400 \mathrm{~K}$ for the larger system. The dotted line shows the conductance of the chain of two atoms. $m$ components as the size of the system increases.

The differences in the curves for the systems of 200 and 300 electrons in Fig. 7 indicate that the finite system size has still some influence. However, there are also clear similarities. For example, there appears shallow conductance minima between deeper minima. This structure is connected to the clustering of the resonance $m=0$ energy levels as seen in the level spectrum at the bottom of Fig. 3. The effect can be considered as reminiscent of the shell structure in the system of conical confinement.

In Fig. 7 we show for the larger system the conductance of the chain of two Na atoms at large cone angles. The conductances of the $N=2$ and $N=3$ chains are in opposite phases and according to our calculations this state continues at least to the angle of $110^{\circ}$. Thus, we were not able to find a limiting blunt angle at which the influence of the resonance states and the even-odd oscillations in the conductance disappear. ${ }^{8,9}$

The oscillation of the conductance as a function of the cone angle means the alternation of perfect electron transport and partial electron scattering in the $m=0$ channel. The electron scattering is caused by the interference between the $m$ $=0$ channel and the nonconducting $m>0$ channel. The situation is analogous to the transport through a quantum wire with a side-coupled quantum dot. ${ }^{15,16}$ If the Kondo resonance of the quantum dot lies at the Fermi level the transport channel DOS at the Fermi level is suppressed (the situation is also called as the Fano antiresonance) and the conductance has a dip. In the present model, the $m>0$ channels play the role of the side-coupled quantum dot.

As discussed in the Introduction, atomic chains have been experimentally observed for Au. ${ }^{3,4}$ These chains have been interpreted to contain up to four Au atoms. It has been noted that before breaking the last conductance plateau of about one conductance quantum is not smooth but exhibits abrupt changes synchronized with abrupt changes in the elongation force. ${ }^{17}$ The steps in the conductance may be of the order of one tenth of the conductance quantum. Our findings give two explanations for these small conductance changes. The conductance may jump abruptly when the number of atoms in the chain increases. On the other hand, the geometry of the lead tips changes affecting also the conductance. These two effects may be superimposed during the actual elongation process.

\section{CONCLUSIONS}

We have studied the electronic properties of chains of $\mathrm{Na}$ atoms between two metallic leads using a pseudoatomjellium model. The conductances of the chains are calculated using the Friedel sum rule. The conductances show even-odd oscillation as function of number of atoms in the chain confirming the earlier results obtained by a purely atomistic model. ${ }^{8}$ There is another oscillation: the conductance oscillates as a function of the lead cone angle. The even-odd oscillation is a direct result of the resonance structure of the electron states of the conducting channel and the charge neutrality at the atom chain and the tips of the leads. The conductance oscillations as a function of the cone angle reflect 
the fact that also electrons of the nonconducting channel take part in the charge neutralization and that the balance between the conducting and nonconducting channel electrons depends on the cone angle. Thus, when determining the conductance of a nanoconstriction it is important to solve self-consistently for the electronic structure, including the lead regions.

\section{ACKNOWLEDGMENTS}

We acknowledge the generous computer resources from the Center for Scientific Computing, Espoo, Finland. This research has been supported by the Academy of Finland through its Centers of Excellence Program (2000-2005).
${ }^{1}$ For a review, see, e.g., C. Joachim, J. K. Gimzewski, and A. Aviram, Nature (London) 408, 541 (2000).

${ }^{2}$ G. Rubio, N. Agrait, and S. Vieira, Phys. Rev. Lett. 76, 2302 (1996).

${ }^{3}$ H. Ohnishi, Y. Kondo, and K. Takayanagi, Nature (London) 395, 780 (1998).

${ }^{4}$ A. I. Yanson, G. Rubio Bollinger, H. E. Van Den Brom, N. Agrait, and J. M. Ruitenbeek, Nature (London) 395, 783 (1998).

${ }^{5}$ R. N. Barnett and U. Landman, Nature (London) 387, 788 (1977).

${ }^{6}$ H. Häkkinen and M. Manninen, Europhys. Lett. 44, 80 (1998).

${ }^{7}$ A. Nakamura, M. Brandbyge, L. B. Hansen, and K. W. Jacobsen, Phys. Rev. Lett. 82, 1538 (1999).

${ }^{8}$ H.-S. Sim, H.-W. Lee, and K.J. Chang, Phys. Rev. Lett. 87, 096803 (2001).

${ }^{9}$ A. L. Yeyati, A. Martín-Rodero, and F. Flores, Phys. Rev. B 56, 10369 (1997).

${ }^{10}$ Norm-conserving nonlocal pseudopotentials are used. N. Troullier and J. L. Martins, Phys. Rev. B 43, 1993 (1991); L. Kleinman and D. M. Bylander, Phys. Rev. Lett. 48, 1425 (1982).

${ }^{11}$ We use the so-called stabilized jellium model introduced in J. P. Perdew, H. Q. Tran, and E. D. Smith, Phys. Rev. B 42, 11627 (1990); H. B. Shore and J. H. Rose, Phys. Rev. Lett. 66, 2519 (1991). The positive background density $n_{+}$corresponds to the average valence electron density of $\mathrm{Na}$ so that the density parameter $r_{s}=3.93 a_{o}\left[n_{+}=3 /\left(4 \pi r s^{3}\right)\right]$.

${ }^{12}$ D. M. Ceperley and B. J. Alder, Phys. Rev. Lett. 45, 566 (1980); J. P. Perdew and A. Zunger, Phys. Rev. B 23, 5048 (1981).

${ }^{13}$ M. Heiskanen, T. Torsti, M. J. Puska, and R. M. Nieminen, Phys. Rev. B 63, 245106 (2001).

${ }^{14}$ S. Datta and W. Tian, Phys. Rev. B 55, R1914 (1997).

${ }^{15}$ K. Kang, S. Y. Cho, J.-J. Kim, and S.-C. Shin, Phys. Rev. B 63, 113304 (2001).

${ }^{16}$ M. E. Torio, K. Hallberg, A. H. Ceccatto, and C. R. Proetto, Phys. Rev. B 65, 085302 (2002).

${ }^{17}$ G. Rubio-Bollinger, S. R. Bahn, N. Agraït, K. W. Jacobsen, and S. Vieira, Phys. Rev. Lett. 87, 026101 (2001). 